\title{
Estimation Method and Simulation of Brightness of Monochromatic LED Point Source Perceived by the Elderly and Protanopes
}

\author{
Taka-aki SUZUKI* and Saki SUKOYAKA** \\ *Industrial Research Institute of Shizuoka Prefecture \\ **TOCLAS CORPORATION
}

Received June 24, 2009, Accepted November 6, 2009

\begin{abstract}
This study aimed at estimating the brightness of a monochromatic LED point source as perceived by the elderly and protanopes. In order to estimate the perceived brightness of the monochromatic LED point source for the elderly and protanopes, we defined an index, brightness perception efficiency, which is a ratio of perceived luminosity with spectral luminous efficiency of the elderly or protanopes to the luminosity with standard spectral luminous efficiency. The brightness perception efficiency was calculated using both the LED spectral radiant intensity defined by a peak wavelength and a full width at half maximum (FWHM) of the LED, and the spectral luminous efficiencies of the elderly in their 70 s and protanopes. The brightness perception efficiency indicated that monochromatic LED point sources with a broad FWHM effectively attenuate the reduction in perceived brightness of the elderly and protanopes. It is expected that in the case of a peak wavelength of $475 \mathrm{~nm}$ the elderly perceive brightness equivalent to 1.6 times luminosity for the LED with a FWHM of $100 \mathrm{~nm}$ compared to that with a FWHM of $15 \mathrm{~nm}$, and in the case of a peak wavelength of $635 \mathrm{~nm}$ protanopes perceived brightness equivalent to about 3 times luminosity for the LED with a FWHM of $100 \mathrm{~nm}$ compared to that with a FWHM of $15 \mathrm{~nm}$. Based on the brightness perception efficiency, we developed an LED illumination simulator that allows the young observers with normal color vision to experience the brightness perceived by the elderly and protanopes.
\end{abstract}

KEYWORDS: elderly peoples, protanope, monochromatic LED, brightness, spectral luminous efficiency

\section{Introduction}

Because of the recent technical innovation of light emitting diodes (LEDs), various types of LEDs are now commercially available, and LEDs are coming into increasingly wider use for control and display equipments. The fundamental function of LEDs on these equipments is to indicate their status (working, waiting, stop, error and so on) by turning on, turning off or blinking the light. Thus, the most basic requirement is that the LED light should be easily recognized by the user. In addition, color universal design, which provides easily visible color display or sign for the elderly and color deficient observers as well as the young with normal color vision, is a desirable feature for interface design of the control and display equipments. However, since monochromatic LEDs emit light in the narrow spectrum range, their perceived brightness varies considerably according to the visual characteristics of individuals. It is known that elderly people perceive a blue LED light to be darker because of age-related changes such as the yellowing crystalline lens in aging vision ${ }^{1)}$, and protanopes perceive a red LED light as darker compared to normal color vision observers. It is also re- ported that individual visual characteristic of the elderly cause a reduction of the visibility for color stimuli, and reaction and recognition times take longer, accordingly ${ }^{233}$. It is therefore important to design operation and display equipment in such a way that LED illumination and blinking is easily visible for elderly and color-deficient users. However, there are not any quantitative evaluation methods of the perceived brightness of monochromatic LEDs for various visual characteristics, considering the effects of peak wavelength and full width at half maximum (FWHM) of the LED. For this reason, system hardware designers did not know, when selecting monochromatic LEDs for use in given products, the optimum LED optical properties including peak wavelength, FWHM and luminosity to make displays easily recognizable for elderly and color deficient users.

In this study, we estimated the reduction in brightness of monochromatic LEDs perceived by the elderly and protanopes based on a peak wavelength and FWHM of a monochromatic LED. We defined brightness perception efficiency as an index of the reduction of the brightness. The brightness perception efficiency 
values were calculated using the peak wavelength and FWHM of the monochromatic LEDs, and the spectral luminous efficiencies of the elderly and protanopes. Furthermore, on the basis of the calculated values, an illumination simulator for the monochromatic LEDs was developed that allows young observers with normal color vision to experience the reduction in brightness perceived by the elderly and protanopes.

Brightness perception is generally estimated using brightness perception efficiency $V_{b}(\lambda)$ measured by the direct comparison method. In past studies, there are reports on the spectral luminous efficiency of dichromats using the flicker method, but no reports on brightness perception efficiency. It is known that if the view size of stimulation is $10 \mathrm{~min}$ or less in visual angle, the spectral luminous efficiency using the flicker method and brightness perception efficiency using the direct comparison method are nearly the same $e^{4)}$.

Therefore, in this study, we considered that the brightness of monochromatic LED point light sources when observed at a view size ${ }^{\dagger}$ of 10 min or less can be estimated using the spectral luminous efficiency obtained by the flicker method, and estimated the brightness. Based on the above, brightness estimation in this study was conducted for monochromatic LED point light sources observed from a view size of $10 \mathrm{~min}$ or less. For view sizes larger than $10 \mathrm{~min}$, monochromatic LEDs blinking in a range of about $10-20 \mathrm{~Hz}$, which is an experimental condition similar to the flicker method, may also be estimated for brightness by this estimation method using spectral luminous efficiency obtained by the flicker method. In addition, monochromatic LED point light sources addressed in this paper have a luminance higher than the background luminance and assume that they are perceived in the light source color mode $^{5}$, in which they appear luminous. In case of the light source color mode, the effects of both the luminance contrast between the light sources and the background, and the adaptation state have little impact on brightness perception. Therefore, this paper does not consider their impact. In addition, this paper discusses brightness perception in a photopic vision.

\section{Formulation of spectral radiant intensity of monochromatic LEDs}

The brightness of a monochromatic LED point source depends largely on its spectral radiant intensity. However, the spectral radiant intensities of LEDs are rarely provided with the products. The provided optical properties of monochromatic LEDs are generally peak

\footnotetext{
$\dagger$ This estimation method cannot be applied to monochromatic LEDs with sustained illumination observed at a view size of more than $10 \mathrm{~min}$, because of the influences of Helmholz-Kohlrausch's effect. Ten minutes in view size represents a size of stimulation (size of light source in this study) that is about $1.5 \mathrm{~mm}$ wide at a $50-\mathrm{cm}$ visual distance, about $3 \mathrm{~mm}$ wide at a $100-\mathrm{cm}$ visual distance, and about $5 \mathrm{~mm}$ wide at a $170-\mathrm{cm}$ visual distance.
}

wavelength, dominant wavelength, FWHM, luminosity and so on. Since the spectral radiant intensity profile of an LED depends on the probability density distribution of the energy gap of an LED, that of the monochromatic LED typically shows a Gaussian-like distribution. It was assumed to be represented by Equation (1) as a Gaussian function having as parameters its peak wavelength and FWHM.

$$
I(\lambda)=\exp \left[-\left(\lambda-\lambda_{p}\right)^{2} / 2 \sigma^{2}\right]
$$

where $I, \lambda$ and $\lambda_{p}$ are spectral radiant intensity, wavelength and peak wavelength of the LED, respectively. FWHM; $\lambda_{p}$ is also determined by $\lambda_{p}=2 \sigma(2 \ln 2)^{0.5}$. Our survey of visible light monochromatic LEDs (ninety eight bullet LEDs of 4 manufacturers) revealed that their peak wavelength values ranged from 430 to 700 $\mathrm{nm}$ and their FWHM ranged from 12 to $100 \mathrm{~nm}$ (Table 1). The following estimations were done for these ranges of peak wavelength and FWHM shown in Table 1.

\section{Spectral luminous efficiency in the elderly and dichromats}

The perceived brightness of monochromatic LEDs depends largely on the individual spectral luminous efficiency. As described in Chapter 1, this study was conducted for estimating the brightness of a monochromatic LED point light source when observed at a view size of 10 min or less using spectral luminous efficiency obtained by the flicker method. Figure 1 shows the spectral luminous efficiencies in the elderly (they were in their 70s, all color normal, and had no ocular damage $)^{6)}$ and dichromatic subjects reported in the previous studies $^{7)-11)}$, and CIE standard spectral luminous efficiency $V(\lambda)$. The spectral luminous efficiencies of the elderly subjects are lower than the other groups at wavelengths shorter than $550 \mathrm{~nm}$, and that of protanopes is the lowest at wavelengths longer than $550 \mathrm{~nm}$. This suggests that it is necessary to pay particular attention to a reduction of the sensitivities in the elderly and protanopes when considering the difference in individual spectral luminance efficiencies. Accordingly, this study deals with the spectral luminous efficiencies of the elderly and protanopes. In order to estimate the perceived brightness of monochromatic LEDs for the elderly and protanopes, we formulated their spectral luminous efficiencies by fitting the data shown in Figure 1 with polynomial equations;

$$
\log \left(V_{\text {elderly }}(\lambda)\right) \text { and } \log \left(V_{\text {protan }}(\lambda)\right)=\sum_{i=0}^{n} a_{i} \lambda^{i}
$$

where $\log \left(V_{\text {elderly }}(\lambda)\right)$ and $\log \left(V_{\text {pro tan }}(\lambda)\right)$ are the spectral luminous efficiencies of the elderly and protanopes, respectively. Figure 2 shows the coefficients of determination for the maximum degree $\mathrm{n}$ of the fitting polynomial 
Table 1 Peak wavelength and full width at half maximum of commercially available monochromatic LEDs

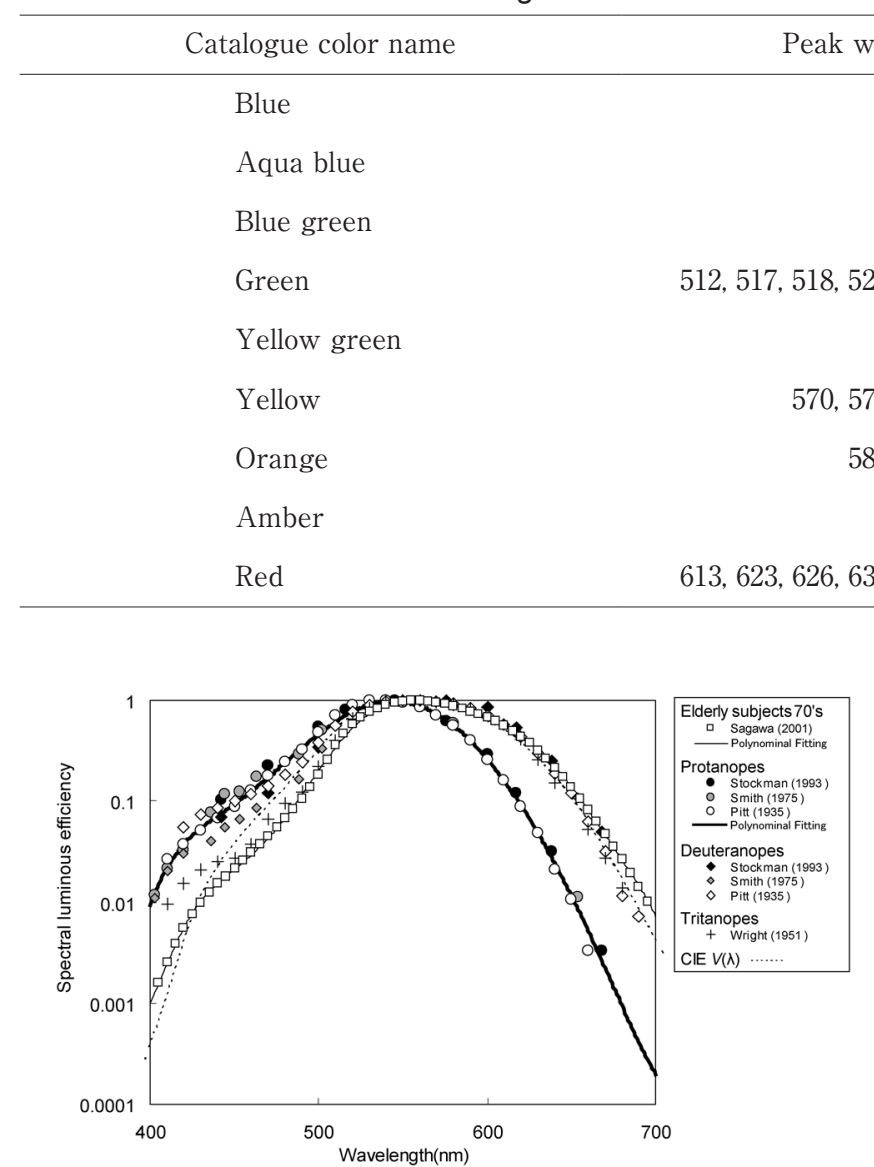

Figure 1 Spectral luminous efficiencies of the elderly, protanopes, deuteranopes, and tritanopes. Solid line shows the fitted polynominal curves of the elderly and protanopes. Dashed line shows $\operatorname{CIE~V~}(\lambda)$.

(2). In Figure 2, it can be seen that the coefficient of determination increases very little with increase of the degree of the polynomial, when the maximum degree $n$ of polynomial is at least 9 for the elderly and at least 7 for protanopes. Therefore, the spectral luminous efficiencies of the elderly and protanopes were fitted with 9th and 7th degree polynomial, respectively. The obtained polynomial coefficients for the elderly and protanopes are shown in Table 2, and the fitted curves are shown as solid lines in Figure 1.

\section{Calculation of brightness perception efficiency}

As an index of the perceived brightness of monochromatic LED point sources for between $V(\lambda)$ and the spectral luminous efficiencies of the elderly or protanopes, we defined with Equation (3), which is a ratio of the perceived luminosity of the elderly or protanopes to the luminosity determined with $V(\lambda)$. We hereinafter refer to this index as "brightness perception efficiency";

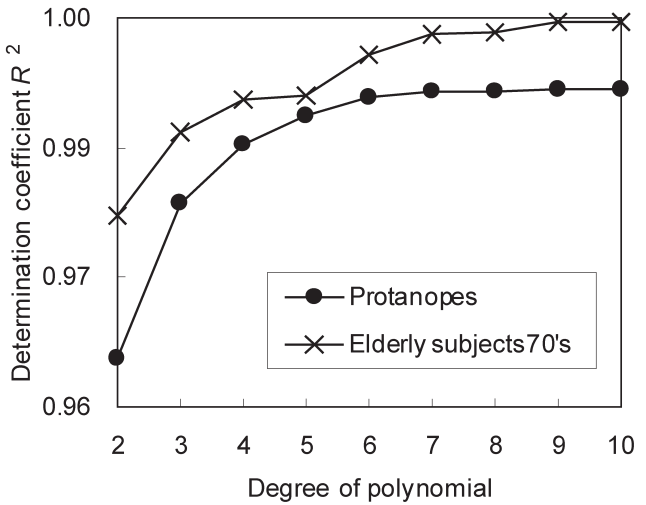

Figure 2 Coefficients of determination for the degree of polynomial fitting of the spectral luminous efficiencies of the elderly and protanopes.

$12,18,20,30,35$

$18,30,40$

$15,17,30,40$

$15,17,19,30,33,40$

$15,16,35$

$15,18,20,25,30,35,40,100$

$$
\frac{K_{m_{-} e} \int_{400}^{700} V_{\text {elderly }}(\lambda) I(\lambda) d \lambda}{K_{m} \int_{400}^{700} V(\lambda) I(\lambda) d \lambda} \text { or } \frac{K_{m_{-} p} \int_{400}^{700} V_{\text {protan }}(\lambda) I(\lambda) d \lambda}{K_{m} \int_{400}^{700} V(\lambda) I(\lambda) d \lambda}
$$

where $k_{m}$ is the maximum luminous efficiency (683 $\mathrm{lm} / \mathrm{W}$ ) established by CIE, and $K_{m_{e} e}$ and $K_{m \_p}$ are maximum luminous efficiencies of the elderly and protanopes, respectively. As stated in the previous chapter, we used spectral luminous efficiency in estimating brightness perception efficiency because spectral luminous efficiency is almost the same as the brightness perception efficiency if the view size of stimulation is 10 min or less in visual angle. Thus, the brightness perception efficiency discussed in this paper will only be suitable for monochromatic LED point light sources observed at a view size of 10 min or less. In other words, the brightness perception efficiency can be regarded as the dimming coefficient indicating by what factor the luminosity of a given monochromatic LED should be 
Table 2 Coefficients for Equation (2)

\begin{tabular}{crr}
\hline $\begin{array}{c}\text { Polynomial } \\
\text { coefficients }\end{array}$ & $\begin{array}{c}\text { Elderly subjects } \\
(70 \text { 's); } \\
\log \left(V_{\text {elderly }}(\lambda)\right)\end{array}$ & $\begin{array}{c}\text { Protanopes; } \\
\log \left(V_{\text {pro tan }}(\lambda)\right)\end{array}$ \\
\hline$a 9$ & $-2.627 \mathrm{E}-19$ & 0 \\
$a 8$ & $1.309 \mathrm{E}-15$ & 0 \\
$a 7$ & $-2.886 \mathrm{E}-12$ & $1.207 \mathrm{E}-15$ \\
$a 6$ & $3.689 \mathrm{E}-09$ & $-4.773 \mathrm{E}-12$ \\
$a 5$ & $-3.016 \mathrm{E}-06$ & $8.055 \mathrm{E}-09$ \\
$a 4$ & $1.634 \mathrm{E}-03$ & $-7.511 \mathrm{E}-06$ \\
$a 3$ & $-5.871 \mathrm{E}-01$ & $4.179 \mathrm{E}-03$ \\
$a 2$ & $1.348 \mathrm{E}+02$ & $-1.386 \mathrm{E}+00$ \\
$a 1$ & $-1.794 \mathrm{E}+04$ & $2.539 \mathrm{E}+02$ \\
$a 0$ & $1.055 \mathrm{E}+06$ & $-1.980 \mathrm{E}+04$ \\
\hline
\end{tabular}

multiplied in order that young observers having $V(\lambda)$ can experience the brightness perceived by the elderly or protanopes seeing the same LED. In this article, young observers with normal color vision are defined as 25-year-old subjects having $V(\lambda)$, because $V(\lambda)$ most closely corresponds to the spectral luminous efficiency at 25 years of age ${ }^{6}$.

To evaluate the brightness perception efficiency of the elderly and protanopes, the maximum luminous efficiency of both visual property groups; $K_{m_{-} e}$ and $K_{m \_p}$ should be determined. For the elderly, Werner et al. ${ }^{12}$ studied age-related change in the absolute threshold for photopic vision for $560 \mathrm{~nm}$ monochromatic light, and reported that the absolute threshold increased with age by a factor of $0.008 A+7.68410$ (expressed in $\log$ quanta $\cdot \mathrm{sec}^{-1} \cdot \mathrm{deg}^{-2}$, and A represents age). Assuming that the young observers are 25 years old and the elderly are 70 years old, the absolute thresholds for the young and the elderly are 7.884 and 8.244 (log quanta $\cdot \mathrm{sec}^{-1} \cdot \mathrm{deg}^{-2}$ ), respectively. This means that the sensitivity of the elderly for $560 \mathrm{~nm}$ light decreases by a factor of $0.437\left(=10^{7.884} / 10^{8.244}\right)$ compared to the sensitivity of the young with normal color vision. In Equation (2), the values of $V_{\text {elderly }}(\lambda)$ and $V(\lambda)$ for $560 \mathrm{~nm}$ light are 0.999 and 0.995 , respectively, and therefore $K_{m_{e} e}=0.437$ -(0.995/0.999) $K_{m}=0.435 K_{m}$. In a study by Werner et al. ${ }^{12)}$, the Maxwell optical system was used in the experiment and the effects of senile miosis were not reflected in the results of the experiment. It should be noted that the effects of senile miosis were not considered in this study. Senile miosis is a phenomenon in which the pupils lose their ability to open, especially in dark places, as a person ages ${ }^{13)}$, and $K_{m_{\_} e}$ may be an even smaller value in dark places. Since the findings obtained in past studies about the effects of senile miosis on brightness perception are insufficient, further studies will also be needed to ascertain the effects of senile miosis on the brightness estimation of monochromatic LEDs, which was currently studied.

In an experiment using a Goldmann-Weekers adaptometer, the absolute threshold for white light for protanopes was reported to be similar to that for trichromats $^{14)}$. Since the Goldmann-Weekers adaptometer uses a tungsten halogen lamp as a white light source, it is inferred that the white light used for the experiment had a spectrum approximate to the spectrum of standard light A. Hence, the following equation was formulated on the assumption that the amount of perceived brightness of standard light $\mathrm{A}$ is equal to the amount of perceived brightness of the white light used for the previous study ${ }^{14)}$

$$
K_{m} \int_{400}^{700} V(\lambda) I_{A}(\lambda) d \lambda=K_{m_{-} p} \int_{400}^{700} V_{p r o t a n}(\lambda) I_{A}(\lambda) d \lambda
$$

where $I_{A}(\lambda)$ represents the relative spectral power distribution of standard light A, as prescribed in CIE Standard: CIE S005. $K_{m \_p}=1.380 K_{m}$ was derived from calculation by substituting $V(\lambda), V_{\text {pro tan }}(\lambda)$ (shown in Equation (2)) and $I_{A}(\lambda)$ in the above equation.

In this paper, which intends to evaluate and estimate the amount of perceived brightness, $K_{m_{-} p}$ was calculated as a coefficient to equalize the amount of brightness perceived by trichromats with the amount of brightness perceived by protanopes. It should be noted that $K_{m_{-} p}$ does not define the maximum luminous efficiency so that the luminous efficiency for $560 \mathrm{~nm}$ assumes a value of $683(\mathrm{~lm} / \mathrm{W})$, unlike $K m$, i.e., the maximum luminous efficiency in terms of scotopic standard spectral luminous efficiency (this also applies to $K_{m \_}$). The result $K_{m \_p}=1.380 K_{m}$ suggests that the sensitivity of protanopes is higher than that of trichromats, which may seem somewhat peculiar. This suggests that protanopes may have the amount of brightness perceived by them compensated for by some perception mechanism, given that the amount of brightness perceived by both protanopes and trichromats is equivalent. In consideration of the fact that the L-cone and M-cone contribute to the brightness perception of trichromats and the fact that the spectral luminous efficiency of protanopes, who lack the L-cone, does not necessarily correspond to the spectral sensitivity of the M-cone, it is inferred that a mechanism different from that of trichromats works in protanopes.

The brightness perception efficiencies of the elderly and protanopes for peak wavelength and the FWHM of a monochromatic LED were calculated using $K_{m_{-},}, K_{m \_-p}$, Equations (1), (2) and (3), and shown in Figure 3 and Figure 4 . In the elderly (Figure 3 ), the brightness perception efficiency for a monochromatic LED at a peak wavelength of $475 \mathrm{~nm}$ and a FWHM of $15 \mathrm{~nm}$ is mini- 


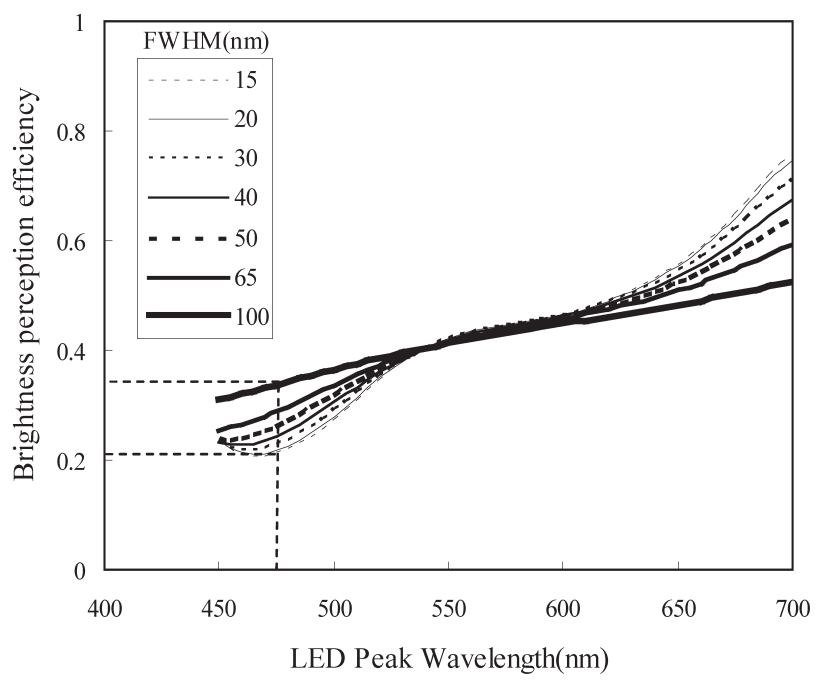

Figure 3 Brightness perception efficiency of the elderly for peak wavelength and FWHM of the monochromatic LED point source.

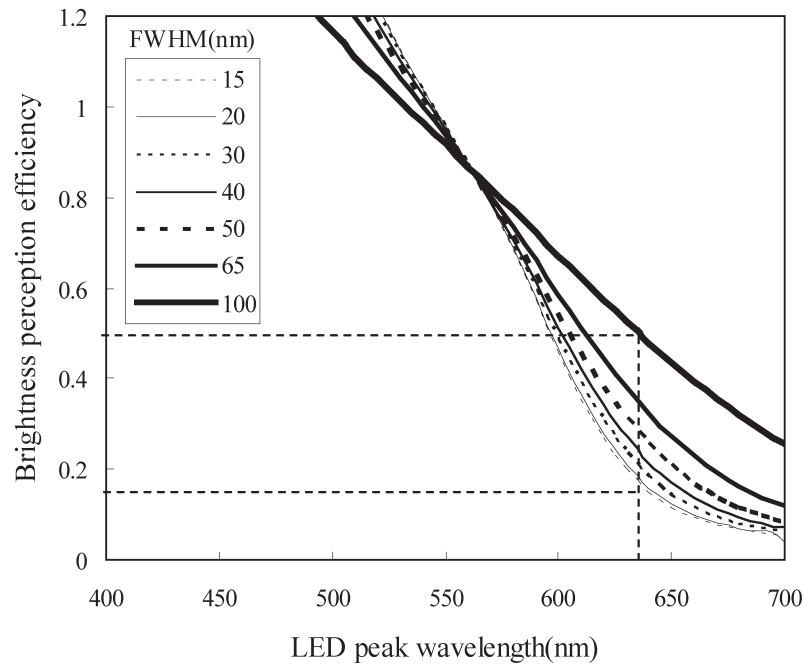

Figure 4 Brightness perception efficiency of protanopes for peak wavelength and FWHM of the monochromatic LED point source.

mum value; 0.212 . This means that the brightness perceived by the elderly is equivalent to the brightness perceived by young observers with normal color vision seeing the same LED with luminosity reduced to its $21 \%$. Even at a peak wavelength of $475 \mathrm{~nm}$, if the FWHM of the monochromatic LED is $100 \mathrm{~nm}$, the brightness perception efficiency is 0.337 , and the reduction in brightness of the monochromatic LED perceived by the elderly will probably be attenuated. In the peak wavelength range of $530-610 \mathrm{~nm}$, even if the FWHM is varied in a range between $15-100 \mathrm{~nm}$, the brightness perception efficiency does not change by more than 0.03. This indicates that in the peak wavelength range between about $530-610 \mathrm{~nm}$ the brightness of the mono- chromatic LED perceived by the elderly does not change for the same peak wavelength even if the FWHM is varied. On the other hand, monochromatic LEDs with a narrow FWHM has larger brightness perception efficiency for peak wavelengths exceeding about $610 \mathrm{~nm}$ and it is expected that the LED will be perceived to be a brighter than LEDs with broader FWHM.

For monochromatic LEDs with a peak wavelength of $700 \mathrm{~nm}$ and a FWHM of $15 \mathrm{~nm}$, the brightness perception efficiency of protanopes is 0.053 (Figure 4). This means that the brightness perceived by protanopes is equivalent to the brightness perceived by young observers with normal color vision seeing the same monochromatic LED luminosity reduced by about $5 \%$. Figure 4 also shows that the reduction in the brightness perception efficiency is attenuated at peak wavelengths longer than $535 \mathrm{~nm}$ by a broader FWHM. For example, in the case of a monochromatic LED with a peak wavelength of $635 \mathrm{~nm}$, the brightness perception efficiency for a FWHM of $15 \mathrm{~nm}$ is 0.229 , while that for a FWHM of $100 \mathrm{~nm}$ is 0.663 . This indicates that even for the same peak wavelength the brightness of an LED perceived by protanopes varies by a factor of three or more according to a broader FWHM, and the broader FWHM will effectively attenuate the reduction in the perceived brightness. In addition, the brightness perception efficiency at peak wavelengths shorter than $535 \mathrm{~nm}$ is more than 1.0 regardless of the FWHM. This is due to the assumption that the absolute threshold of protanopes for white light is similar to that of observers with normal color vision. On this assumption, it is expected that the sensitivity to short wavelengths is augmented to compensation for the reduction in sensitivity to long wavelengths, and protanopes might perceive shortwavelength LED point sources to be brighter than standard observers. The brightness perception efficiency for peak wavelength and the FWHM suggest that the visibility for the elderly and protanopes can be improved by considering FWHM as well as peak wavelength when adopting monochromatic LEDs for a product's display. In the current study, the spectral radiant intensity profile of monochromatic LED point light sources was defined by the Gaussian function in Equation (2). Even if the spectral radiant intensity profile is not a Gaussian function but can be defined, brightness perception efficiency can be calculated. The result indicating that brightness perception efficiency becomes less dependent on wavelength as FWHM becomes wider might be considered in some sense to be a matter of course. Until now, however, it has been impossible for designers selecting monochromatic LED point light sources to numerically estimate reductions in the brightness perception of elderly people and protanopes from the peak wavelength (i.e., color) shown in the cata- 
$\log$ sheets for such LEDs and/or FWHM. In addition, LEDs with a wider FWHM cannot emit the high chroma light, and adopting those LEDs for any colors leads to a reduction in the freedom of color design. It is believed that the utilization of the results obtained from the recent study will make it possible to find an appropriate solution for two contradictory design requirements, namely, the employment of high-chroma LEDs and improvement in brightness perception.

\section{Simulation of the brightness of monochromatic led point source perceived by the elderly and protanopes}

Using obtained brightness perception efficiency values, it is possible to evaluate how dark (or bright) the elderly and protanopes perceive monochromatic LED point sources numerically. However, when adopting appropriate LEDs for control and display equipments, there is a need to confirm if the monochromatic LEDs have the least necessary level of brightness for adequate visibility by a visual evaluation. For this reason, we proposed a simulation system that allows young observers with normal color vision to experience the brightness perceived by the elderly and protanopes when seeing same monochromatic LEDs on the basis of the brightness perception efficiency. Figure 5 illustrates a schematic diagram of this system. The peak wavelength and FWHM of the monochromatic LED and the simulating object group (the elderly or protanopes) are input into a $\mathrm{PC}$. The $\mathrm{PC}$ calculates the brightness perception efficiency from the input parameters, and sends control signals to a computer board to turn on the LED with the luminosity multiplied by the brightness perception efficiency. The computer board generates the pulse width modulated rectangular waves $(1.56 \mathrm{MHz}$ frequency) and controls luminosity of the LEDs by changing the ON-time duration of the pulse. As shown in Figure 5, two same target monochromatic LEDs put on this system. One is used as a reference LED, and another is turned on with a luminosity equivalent to that of the reference LED multiplied by the brightness perception efficiency. In this way, young observer, i.e. young designers, with normal color vision can visually verify the level of brightness of the monochromatic LED as perceived to be by the elderly and protanopes compared to the brightness perceived the reference LED. As stated in Chapter 1, brightness estimation in this study was conducted for monochromatic LED point light sources with sustained illumination when observed at a view size of 10 min or less. Thus, when the brightness of monochromatic LED point sources with sustained illumination in this simulation is evaluated, they should be observed in a condition of $10 \mathrm{~min}$ or less in LED view size $(104 \mathrm{~cm}$ or more in viewing distance for $3-\mathrm{mm}$ diameter LEDs and $172 \mathrm{~cm}$ or more in viewing distance for 5 -mm diameter LEDs). Figure 6 shows the result of a simulation of the reduced brightness perceived by the elderly for a blue LED. Figure 6 shows a simulation result of the reduction in brightness of a blue LED (peak wavelength 470nm, FWHM $30 \mathrm{~nm}$ ) perceived by the elderly. The brightness perception efficiency for the elderly of this LED was 0.227 .

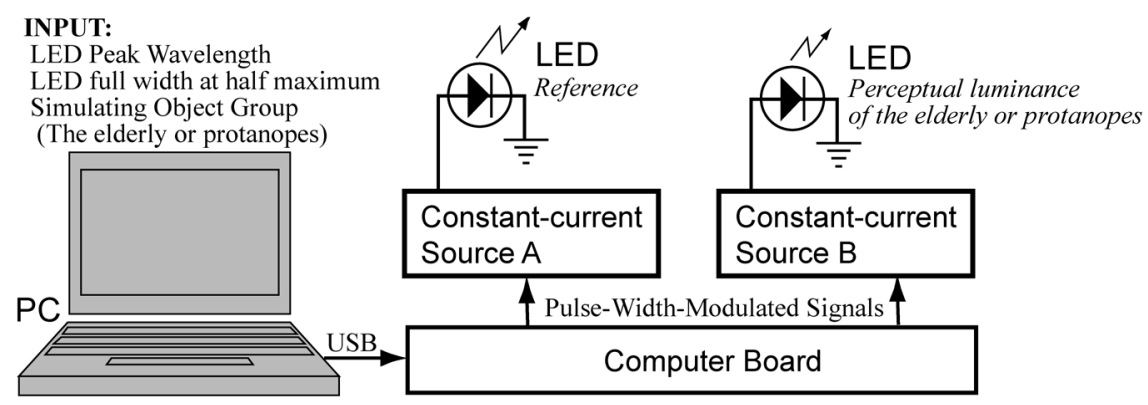

Figure 5 Representation of the simulation system for monochromatic LED brightness.

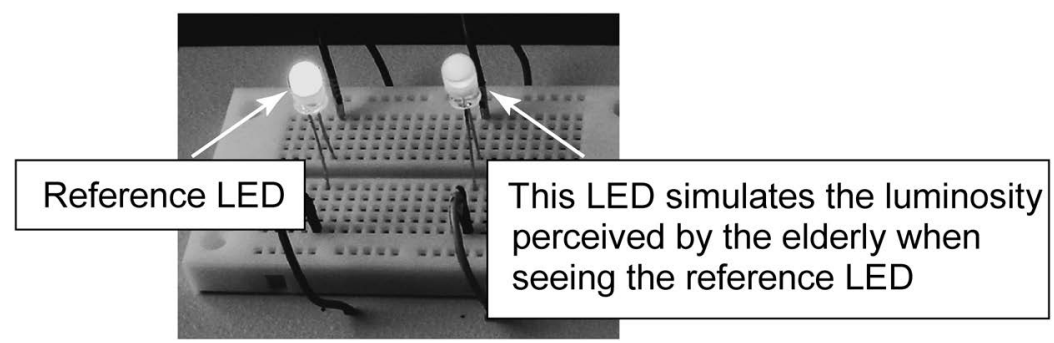

Figure 6 Simulation of the brightness perceived by elderly subjects for a blue LED (peak wavelength $470 \mathrm{~nm}, \mathrm{FWHM} 30 \mathrm{~nm}$ ). 


\section{Conclusion}

In this study, "brightness perception efficiency" was defined as an index that represents the reduction in brightness perceived by the elderly and protanopes when observing monochromatic LED point light sources at a view size of $10 \mathrm{~min}$ or less, and the brightness perception efficiency were estimated for each peak wavelength and FWHM of monochromatic LEDs. The results confirmed numerically that the reduction in brightness of the monochromatic LEDs perceived by the elderly and protanopes can be attenuated by changing the FWHM for LEDs with the same peak wavelength. In addition, we developed an LED illumination simulator that allows young designer with normal color vision to experience the reduction in brightness perceived by the elderly and protanopes on the basis of the brightness perception efficiency. These results can be applied to the assessment of LEDs so that a user friendly design suited to the elderly and protanopes can be achieved. Because the current estimation method uses spectral luminous efficiency obtained by the flicker method, as in the flicker method, the brightness of monochromatic LEDs blinking in a range of about 10-20 $\mathrm{Hz}$, may be estimated by this method even if the view size is $10 \mathrm{~min}$ or more. Furthermore, these results and the evaluation method can be used to assess the spectral radiant intensity when developing new LEDs to ensure good visibility for the elderly and protanopes.

\section{Acknowledgements}

The authors would like to thank Mr. Toshiki YAMASHITA at TOCLAS CORPORATION for his suggestions related to the direction of this study.

\section{References}

(1) Suzuki, T., Okada, Y., Yi, Q., Sakuragawa, S., Takayama, K. and Okajima, K.: Simple estimation method for age-related change of spectral luminous efficiency using LEDs, Proc. AIC2008, Paper no 038 (2008).

(2) Suzuki, T., Yi, Q., Sakuragawa, S., Tamura, H. and Okajima, K.: Comparing the visibility of low-contrast color landolt cs.-Effect of aging human lens -, Color Res. Appl., 30-1, pp. 5-12 (2005).
(3) Suzuki, T., Yi, Q., Sakuragawa, S., Tamura, H. and Okajima, K.: Age-related changes of reaction time and P300 for low-contrast color stimuli: Effects of yellowing of the aging human lens, J. Physiol. Anthropol. Appl. Human Sci., 25-2, pp. 179-187 (2006).

(4) Ikeda, M., Yaguchi, H. and Ohmi, M.: Luminous efficiency function for small stimulus size, J. Light Vis. Env., 5, pp. 25-27 (1981).

(5) Uchikawa, H., Uchikawa, K. and Boynton, R. M.: Influence of achromatic surrounds on categorical perception of surface colors, Vision Res., 29, pp. 881-890 (1984).

(6) Sagawa, K. and Takahashi, Y.: Spectral luminous efficiency as a function of age, J. Opt. Soc. Am.(A), 18, pp. 2659-2667 (2001).

(7) Stockman, A., MacLeod, D. I. and Johnson, N. E.: Spectral sensitivities of the human cones, J. Opt. Soc. Am.(A), 10, pp. 2491-2521 (1993).

(8) Smith, V. C. and Pokorny, J.: Spectral sensitivity of the foveal cone photopigments between 400 and 500 nm, Vision Res., 15, pp. 161-171 (1975).

(9) Hsia, Y. and Graham, C. H.: Spectral luminosity curves for protanopic, deuteranopic, and normal subjects, Proc. Natl. Acad. Sci. U.S.A., 43, pp. 10111019 (1957).

(10) Wyszecki, G. and Stiles, W. S.: Color Science 2nd Edition., Wiley, NewYork (1982).

(11) Wright, W. D.: The characteristics of tritanopia, J. Opt. Soc. Am., 42, pp. 509-521 (1952).

(12) Werner, J. S., Schelble, K. A. and Bieber, M. L.: Agerelated increases in photopic increment thresholds are not due to an elevation in intrinsic noise, Color Res. Appl., 26-S1, pp. S48-S52 (2001).

(13) Suzuki, T., Okajima, K. and Funai, T.: Optical simulation of reduced retinal illuminance caused by senile miosis, Optical Review, 19-3, pp. 174-181 (2012).

(14) Krill, A. E. and Beutler, E.: The red-light absolute threshold in heterozygote protan carriers; Possible genetic implications, Investigative Ophthalmology and Visual Science, 3, pp. 107-118 (1964).

Paper originally published in Japanese in J. IEIJ, Vol. 94 No. 2, 2010

The affiliation of Saki SUKOYAKA is Panasonic Corporation in currency. 